\title{
Simulated annealing with constraints aggregation for control of the multistage processes
}

\author{
Paweł Drạg \\ Department of Control Systems and Mechatronics \\ Wrocław University of Technology \\ Janiszewskiego 11-17, 50-372 Wrocław, Poland \\ Email: pawel.drag@pwr.edu.pl
}

\author{
Krystyn Styczeń \\ Department of Control Systems and Mechatronics, \\ Wrocław University of Technology \\ Janiszewskiego 11-17, 50-372 Wrocław, Poland \\ Email: krystyn.styczen@pwr.edu.pl
}

\begin{abstract}
In the article the control and optimization of multistage technological processes were discussed. In the presented research, it was assumed, that in the complex technological processes, the multistage differential-algebraic constraints with unknown consistent initial conditions were considered. To rewrite the infinite-dimensional optimal control problem into the finitedimensional optimization task, the direct shooting method was applied. Simulated annealing algorithm was proposed as the method for solving nonlinear optimization problem with constraints. Stretching function was used to allow us to locate the globally optimal solution. The complex process constraints were treated using constraints aggregation methods. The presented methodology was tested with optimal control problem of the two-reactors system. The numerical simulations were executed in MATLAB environment using Wroclaw Center for Networking and Supercomputing.

Index Terms - optimal control, DAE systems, simulated anneal-
\end{abstract} ing, constraints aggregation, stretching function.

\section{INTRODUCTION}

$\mathbf{N}$ OWADAYS, technological processes have been modeled using more general and complex systems of equations. To model technological processes in such branch of industry, like chemical engineering, biotechnology and aerospace engineering, both dynamics and physical conservation laws have been under consideration [2], [3], [8], [16]. Then, a validated model of the process can be applied to control and optimize the technological systems. In the other words, to ensure an efficient and trouble free technological processes, an optimization problem subject to nonlinear differentialalgebraic constraints need to be solved [4], [13].

In the last years, deterministic, as well as stochastic optimization algorithms, are adjusted to solve new advanced technological problems. New methods in modeling, algorithmic procedures and globalization of the obtained solutions can be observed in all areas of optimization [9], [10], [26]. In the presented work we would like to indicate the simulated annealing algorithm, which has been seen as one of the main global optimization procedures. In this work the new aspects of the simulated annealing algorithm were given and discussed.

Direct shooting method enables us to transform the optimal control problem into medium- or large-scale nonlinear optimization problem. Then, using aggregation and disaggregation procedures, the considered model can be represented by other system, which reflects the most important features of the original process, but is better adjusted for computational optimization methods [14]. Then, the obtained results can be applied to control the original system.

The presentation of new methodology was performed in the following way. In the next section optimal control with differential-algebraic constraints was introduced. Then, in section III, optimization with aggregated constraints was discussed. "Stretching" function technique in optimization procedures was presented in section IV. Then, a new simulated annealing algorithm with constraints was given and tested in sections IV and V. The considerations were concluded in section VI.

\section{OPTIMAL CONTROL WITH DIFFERENTIAL-ALGEBRAIC CONSTRAINTS}

The optimal control algorithms are highly connected with the available modeling methods. The main objective of the control is always the same - we need to find the control function, which optimizes one of the known forms of the process performance index. But the assumptions, conditions and complexity of the considered processes are increasingly challenging and difficult to solve.

In the last years, new particular types of systems have become popular due to many practical applications. There are the multistage dynamical systems and processes with differential-algebraic equations, which are more general, than the pure dynamical processes.

The most important common features of the systems mentioned above, is the presence of the dynamics in the model. The difference, which has a far-reaching consequences in the calculation methods comes from the non-dynamical part of the model.

In the multistage processes, the state variables have to be continuous across the stages. It means, that in the known points, at the interface between the processes, algebraic continuity constraints are introduced. Therefore, the additional algebraic constraints have a pointwise character.

In processes with differential-algebraic constraints, the algebraic relations are justified by physical laws, which take place in the process and are reflected in the model. In this way, both the differential and algebraic equations have a continuous 
character and can be treated as a one differential-algebraic system [6].

The presented work concerns the case, when the process is multistage and each stage was described using its own system of differential-algebraic equations. In this way, new theoretical assumptions have to be made and new computational concepts should be designed and implemented.

The features presented above lead us to the optimal control problems in the known form. At first, the process performance index, which can be treated as the measure of the control quality, has to be defined as follows

$\min _{u(t)} Q=\int_{0}^{t_{F}} \mathcal{L}(y(t), z(t), u(t), p, t) d t+\mathcal{E}\left(y\left(t_{F}\right), z\left(t_{F}\right), t_{F}\right)$

with single system of differential-algebraic constraints in semiexplicit form

$$
\begin{aligned}
B(t) \dot{y}(t) & =f(y(t), z(t), u(t), p, t) \\
0 & =g(y(t), z(t), u(t), p, t),
\end{aligned}
$$

where $y(t) \in \mathcal{R}^{n_{y}}$ is a differential state, $z(t) \in \mathcal{R}^{n_{z}}$ is an algebraic state and $u(t) \in \mathcal{R}^{n_{u}}$ denotes the unknown control function. The independent variable (e.g. time or length of the chemical reactor) is denoted as $t \in \mathcal{R}$. As $p \in \mathcal{R}^{n_{p}}$ was denoted the vector of global parameters constant in time. The variables in the process are defined by vector-valued functions:

$$
f: \mathcal{R}^{n_{y}} \times \mathcal{R}^{n_{z}} \times \mathcal{R}^{n_{u}} \times \mathcal{R}^{n_{p}} \times \mathcal{R} \rightarrow \mathcal{R}^{n_{y}}
$$

and

$$
g: \mathcal{R}^{n_{y}} \times \mathcal{R}^{n_{z}} \times \mathcal{R}^{n_{u}} \times \mathcal{R}^{n_{p}} \times \mathcal{R} \rightarrow \mathcal{R}^{n_{z}} .
$$

To ensure, that the system (2) consists of differentialalgebraic equations in semi-explicit form, it is assumed, that the matrix $B(t)$ is invertible for all values of $t$.

As it was mentioned, the quality of the process is measured by the value of $Q \in \mathcal{R}$. It is an important assumption, that quality of the whole complex process can be specified by only one real number.

When the multistage processes are considered, then each stage can be described using its own system of differentialalgebraic equations

$$
\begin{aligned}
B^{i}(t) \dot{y}^{i}(t)= & f^{i}\left(y^{i}(t), z^{i}(t), u^{i}(t), p, t\right) \\
0= & g^{i}\left(y^{i}(t), z^{i}(t), u^{i}(t), p, t\right), \\
& i=1, \cdots, N S
\end{aligned}
$$

where $N S$ denotes number of the stages in the considered process.

One of the most important progresses in the optimal control methods has been connected with parametrization of the control problem. Because in this way the optimal control problem can be treated as a nonlinear optimization problem, efficient numerical optimization algorithms can be applied.

The parametrization of the control function has been proposed in the article [24] in 1994. Today, the piecewise continuous control parametrization using constant, linear or quadratic functions has been a commonly used approach.
In this way, instead to search for optimal solution using advanced analytical methods, the efficient algorithm of numerical optimization can be applied to obtain the best solution in the assumed class of piecewise continuous functions [7].

The parametrization of the control function in optimal control problems has been often used together with the multiple shooting technique. The multiple shooting method is appropriate to decompose the complex technological processes as well as to stabilize the solutions of both the unstable and highly nonlinear systems.

The multiple shooting method together with the control function parametrization lead us to the direct shooting approach. In this methodology, at the first step, the independent variable domain is divided into the assumed number of intervals

$$
t=\bigcup_{i=1}^{N S-1}\left[\begin{array}{ll}
t_{i-1} & t_{i}
\end{array}\right) \cup\left[\begin{array}{ll}
t_{N S-1} & t_{N S}
\end{array}\right],
$$

where $t_{0}$ is the time instant, when the process starts and $t_{N S}$ is the final time.

Therefore, the control function as well as the differentialalgebraic system can be parametrized in each interval. In practical applications, when the control function is parametrized as piecewise constant, then

$$
u^{i}(t)=u_{i}, \quad i=1, \cdots, N S .
$$

The differential-algebraic model of the technological process is parametrized in the sense of the unknown initial conditions. This approach enables us to solve the DAE system using the efficient numerical procedures [17]. The initial conditions of differential-algebraic system can be parametrized in the following way

$$
\begin{aligned}
& y^{i}\left(t_{i-1}\right)=s_{y}^{i} \\
& z^{i}\left(t_{i-1}\right)=s^{i}
\end{aligned}
$$

for $i=1, \cdots, N S$.

Therefore, the differential-algebraic constraints have been obtain the new form

$$
\begin{aligned}
B^{i}(t) \dot{y}^{i}(t) & =f^{i}\left(y^{i}(t), z^{i}(t), u^{i}, p, t^{i}\right) \\
0 & =g^{i}\left(y^{i}(t), z^{i}(t), u^{i}, p, t^{i}\right),
\end{aligned}
$$

with $t^{i} \in\left[\begin{array}{ll}t_{i-1} & t_{i}\end{array}\right]$ and $i=1, \cdots, N S$. Because the consistent initial conditions are needed to solve the DAE systems, the algebraic part can be extended by damping factor $\alpha$

$$
0=g^{i}\left(y^{i}(t), z^{i}(t), u^{i}, p, t^{i}\right)+\alpha^{i}(t) g^{i}\left(s_{y}^{i}, s_{z}^{i}, u^{i}, p, t^{i}\right)
$$

for $t^{i} \in\left[\begin{array}{ll}t_{i-1} & t_{i}\end{array}\right]$ and $i=1, \cdots, N S$.

The process performance index has been rewritten as follows

$\min _{s_{y}^{i}, s_{z}^{i}, u^{i}} \hat{Q}=\sum_{i=1}^{N S} \int_{t_{i-1}}^{t_{i}} \mathcal{L}\left(s_{y}^{i}, s_{z}^{i}, u^{i}, p, t\right) d t+\mathcal{E}\left(s_{y}^{N S}, s_{z}^{N S}, t_{F}\right)$.

It is worth to note, that the trajectories of the differential states are continuous. It means, that the additional relations have to be incorporated to the model

$$
y^{i}\left(t_{i}\right)=y^{i+1}\left(t_{i}\right)=s_{y}^{i+1}, \quad i=1, \cdots, N S-1,
$$


which results as additional pointwise algebraic constraints of the following form

$$
y^{i}\left(t_{i}\right)-s_{y}^{i+1}=0, \quad i=1, \cdots, N S-1 .
$$

In the most practical situations, the unknown parameters have their physical interpretation. Among them can be distinguished an unknown volume of the chemical reactor, concentration of the substrates or value of the temperature. The interpretation of the decision variables and technological restrictions defines the feasible region and can be rewritten as the inequality constraints in the form of lower and upper bounds.

Direct shooting approach enables us to transform the optimal control problem into the nonlinear programming problem with the vector of decision variables designed as

$$
x=\left[\begin{array}{llll}
s_{y}^{i} & s_{z}^{i} & u_{i} & p
\end{array}\right]^{T},
$$

the process performance index in the form of the objective function

$$
\min _{x} \hat{Q}(x)
$$

differential-algebraic constraints

$$
\begin{aligned}
B^{i}(t) \dot{y}^{i}(t) & =f^{i}\left(y^{i}(t), z^{i}(t), u^{i}, p, t^{i}\right) \\
0 & =g^{i}\left(y^{i}(t), z^{i}(t), u^{i}, p, t^{i}\right),
\end{aligned}
$$

consistency constraints

$$
0=g^{i}\left(y^{i}(t), z^{i}(t), u^{i}, p, t^{i}\right)+\alpha^{i}(t) g^{i}\left(s_{y}^{i}, s_{z}^{i}, u^{i}, p, t^{i}\right)
$$

continuity constraints

$$
y^{i}\left(t_{i}\right)-s_{y}^{i+1}=0,
$$

and the constraints of upper and lower bounds type

$$
x_{L} \leq x \leq x_{U}
$$

for $t^{i} \in\left[\begin{array}{ll}t_{i-1} & t_{i}\end{array}\right]$ and $i=1, \cdots, N S$.

This causes, that the various numerical optimization algorithms can be treated as an important part of the control algorithms [18]. This transformation is a main effect of the direct shooting method applied in the optimal control problems.

\section{OPTIMIZATION WITH AGGREGATED CONSTRAINTS}

The complexity of the considered models and large number of decision variables makes, that the analytical solutions and some intuitive numerical approaches can be inappropriate in the control of the real-life technological processes.

One of the most important question in modern optimization and control theory is, how to solve the problems with thousands or millions variables and comparable number of equality and inequality constraints. The second important question is, how to compare two unfeasible solutions and to make a decision, which one proposed solution is better than the others.

To analyze the complex process, both the aggregation and disaggregation techniques were used. There is a general methodology, how to obtain useful information about the process by a possibly little model modifications and computations amount [5].
In the previous section, the optimal control problem was reformulated as a middle- or large-scale nonlinear optimization problem. To do this, the direct shooting method was applied. At this point, the consecutive question cannot more wait for the answer - how to treat optimization problems with so many variables and subject to huge number of constraints? The question, which has been just imposed, is considered in this section.

The general way to obtain the useful information about the complex process can be constructed as follows [23]

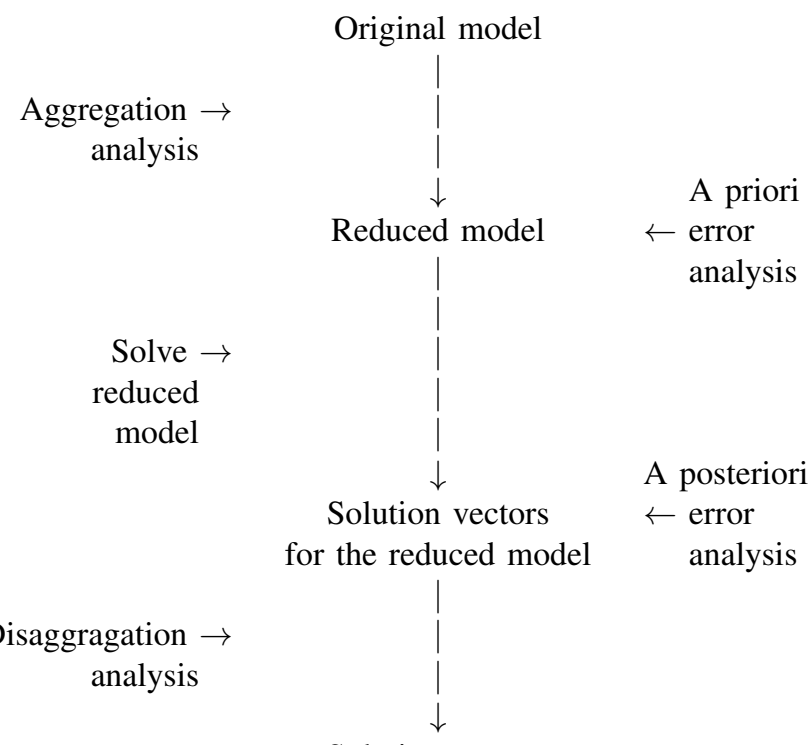

Solution vectors

for the original model
Solve for $\rightarrow$ original objective function

Estimate of

objective function value for original model

In a general sense, in model aggregation, large-scale timization models are reformulated as less complex systems. The obtained in this way models reflect the most important features of the original systems, but should be much more suitable to perform numerical simulations.

In the other words, the aggregation technique is a method to specified parts of the model, which can be described using only one single element. At this step, the new single element should be explicitly defined. Therefore, aggregation analysis consists of two levels:

- process of determining, which data are in some sense similar and can be considered as elements in the same group; this step is known as cluster analysis,

- the method of combining the clustered data to define the reduced model. 
In contrast to the aggregation analysis, the disaggregation is a method, which can derive the information about the complex model using results obtained by reduced system analysis. The disaggregation analysis, often known as the reversal aggregation analysis, enables us to estimate the solution of the original system using only results obtained from solving the reduced model.

The last stage, which is connected with aggregation and disaggregation methodology, is an error analysis. The error analysis determines the resulting error, which can be introduced by algorithms with aggregate models and disaggregate solutions. From practical point of view, the error analysis can be useful for selection of appropriate aggregation and disaggregation procedures. In the literature two main types of error are defined

a) a priori error bounds, which are the bounds placed upon the optimal value of an original optimization model after aggregation stage, but before the aggregate model is solved,

b) a posteriori error bounds are the bounds placed upon the optimal value of an original optimization model after the aggregated model has been formed and solved.

In practical applications, the iterative optimization algorithms with successive aggregation-disaggregation schemes are used. Such procedures are known as an Iterative AggregationDisaggregation (IAD) technique.

As it was mentioned, the direct shooting method allows us to transform the optimal control problem into a nonlinear optimization problem. Therefore, solution of the original infinite-dimensionally task can be obtained by solving finitedimensional optimization problem. But the question posed in this section does not refer to the optimal control problems. It concerns the much more fundamental task - how to solve medium- and large-scale optimization problems?

It is expected, that the efficient numerical optimization methods enable us to solve optimal control problems.

Therefore, we would like to propose aggregation methods, which can be useful to define new reduced process performance index, as well as reduced constraints.

At this place, we would like to focus on the constraints function aggregation. It was mentioned, that two main types of the constraints need to be considered in the process - there are continuous and pointwise constraints. In particular, it means, that some group of functions are connected with process description by continuous differential-algebraic constraints There is the second group of constraints, which denotes the additional constraints at the prescribed time instances in the process.

Let us consider the system of the continuous differentialalgebraic equations. This particular system can be solved by implementation of efficient numerical algorithms. After that, the process performance index, as well as fulfillment of the constraint can be determined. There is an important question, which refers to consistent initial conditions, which enables us to solve the differential-algebraic system. Although the initial conditions are defined at the specific point, they strictly refer to the continuous process constraints and should be perceived with continuous differential-algebraic constraints. In this work, we refer to this type of constraints as the consistency constraints and denote as

$$
\begin{aligned}
c_{\text {cons }}( & \left.\left\{s_{y}^{i}, s_{z}^{i}, u^{i}, p, t_{i-1}\right\}_{i=1}^{N S}\right)= \\
= & {\left[\begin{array}{c}
g^{1}\left(s_{y}^{1}, s_{z}^{1}, u^{1}, p, t_{0}\right) \\
g^{2}\left(s_{y}^{2}, s_{z}^{2}, u^{2}, p, t_{1}\right) \\
\vdots \\
g^{N S}\left(s_{y}^{N S}, s_{z}^{N S}, u^{N S}, p, t_{N S-1}\right)
\end{array}\right]=0 . }
\end{aligned}
$$

The other group of constraints represents the pointwise constraints, which are connected with the fact, that the multistage system is considered. In the other words it means, that the differential state trajectories are continuous across the process stages. The vector of pointwise algebraic constraints denoted as $c_{\text {cont }}$ is defined as follows

$$
\begin{gathered}
c_{\text {cont }}\left(\left\{s_{y}^{i+1}, s_{z}^{i+1}, u^{i}, p, t_{i}\right\}_{i=1}^{N S-1}\right)= \\
=\left[\begin{array}{c}
y^{1}\left(t_{1}\right)-s_{y}^{2} \\
y^{2}\left(t_{2}\right)-s_{y}^{3} \\
\vdots \\
y^{N S-1}\left(t_{N S-1}\right)-s_{y}^{N S}
\end{array}\right]=0 .
\end{gathered}
$$

In some numerical optimization algorithms, e.g. Sequential Quadratic Programming, it is suggested to treat each constraint function in the same way. Therefore, in each iteration, a largescale and possibly structured matrix of constraint functions derivatives can be obtained. In general case, when the structure of the process constraints is unknown, the matrix of partial derivatives of the constraints might be time and memory consuming.

If, in general, $c(x)$ denotes

$$
c(x)=\left[\begin{array}{c}
c_{1}(x) \\
c_{2}(x) \\
\vdots \\
c_{N S}(x)
\end{array}\right]=0
$$

then the vector of the constraint functions can be replaced using one of the following real-valued aggregated functions

$$
\begin{gathered}
\tilde{c}(x)=\|c(x)\|_{1}, \\
\tilde{c}(x)=\|c(x)\|_{2}
\end{gathered}
$$

or

$$
\tilde{c}(x)=\|c(x)\|_{\infty} .
$$

One cannot determine, which one measure of the constraints infeasibility has the best features. The constraints aggregation function can be chosen depending on the considered process, optimization methods and, first of all, available computing resources.

The other question is, how to treat the process performance index. Parametrization of the control function, as well as differential and algebraic states, enables us to rewrite the 
process performance index as the objective function in nonlinear optimization problem. In some applications the penalty function approach and many variations of it can be observed. Advanced optimization methods focus on methods without penalty function [12], [22]. For these reasons, in presented methodology, the objective function $\hat{Q}(x)$, which represents control quality index, was remained unchanged.

\section{STRETCHING FUNCTION}

In the previous section it has been indicated, how to apply the direct shooting method to transform the optimal control problem into the nonlinear optimization task. In general, the presented methodology enables us to consider the optimization problem over the feasible region

$$
\min _{x} f(x), \quad \forall x \in \mathcal{A},
$$

where $f(x)$ is a real-valued objective function

$$
f: \mathcal{A} \rightarrow \mathcal{R}
$$

where $\mathcal{A} \subseteq \mathcal{R}^{D}$ is the D-dimensional compact set.

The important question is, how to treat the nonlinear optimization problem with the large number of equality, as well as inequality constraints.

To solve the optimization problem on the compact set, the "stretching" technique for the objective function can be applied [19].

Let us consider the objective function $f(x)$ defined on the compact set. Let the objective function $f$ get the local minimum in a point $x^{\star}$. Therefore, the point $x^{\star}$ is a local minimizer of the function $f$. As a consequence, it means, that in the neighborhood $\mathcal{N B}$ of the point $x^{\star}$, the inequality

$$
f\left(x^{\star}\right) \leq f(x)
$$

is satisfied for all $x \in \mathcal{N B}$.

In real-life optimization and control problems, it is highly undesirable, that one of the low quality local minima can be treated as the global solution. The "stretching" function method was designed as a remedy for described situation. This method enables us to alleviate the local minimum using the following two-step transformation.

Let $x^{\hat{\star}}$ be the local minimum of the function $f$, then in the step 1 , the function $f(x)$ elevates and all the local minima, which are above the point $f\left(x^{\hat{\star}}\right)$, disappear

$$
\mathcal{G}(x)=f(x)+\frac{\gamma_{1}}{2}\left\|x-x^{\hat{\star}}\right\|\left(\operatorname{sign}\left(f(x)-f\left(x^{\hat{\star}}\right)\right)+1\right)
$$

In the step 2, the neighborhood of the point $x^{\hat{\star}}$ is stretched upwards, in this way, that it assigns higher values of those points

$$
\begin{aligned}
\mathcal{S}(f(x)) & =f^{\mathcal{S}}(x)=\mathcal{H}(x)= \\
= & \mathcal{G}(x)+\frac{\gamma_{2}\left(\operatorname{sign}\left(f(x)-f\left(x^{\hat{\star}}\right)\right)+1\right)}{2 \tanh \left(\mu\left(\mathcal{G}(x)-\mathcal{G}\left(x^{\hat{\star}}\right)\right)\right)} .
\end{aligned}
$$

The parameters $\gamma_{1}, \gamma_{2}$ and $\mu$ are the positive constants with arbitrary chosen value. The authors, who proposed the "stretching" function technique as the global optimization method, suggested the values of the mentioned parameters as $\gamma_{1}=10000, \gamma_{2}=1, \mu=10^{-10}$. It was suggested, that the choice of the considered parameters seems not to be critical for the success of the optimization, but they can influence the convergence rate. For these reasons, the parameter tuning was suggested [20].

It is an important question, how the "stretching" function technique can be applied for optimization problems, when a feasible region is no more a compact set? The mentioned situation can be meet in all cases, when optimization problems with equality constraint are considered [21].

To find the answer for the question posed above, the constraints aggregation methodology is very helpful.

\section{Simulated ANNEALING With CONSTRAints}

The main ideas connected with simulated annealing algorithm have their origin in thermodynamics. The physical laws, which governs cooling of molten metal in annealing process, have been used to design new optimization methods. After slow cooling in annealing process, the metal tends to reach a state with a low energy. In general, the state with the minimum energy is desired. In analogy to annealing process, the energy represents the possible solution of the optimization problem. Therefore, the state with the minimal energy represents the solution in the optimization algorithm [15], [25].

The simulated annealing algorithm is constructed as follows.

\section{The simulated annealing algorithm}

$$
\begin{aligned}
& \text { Initialization }\left(x_{0}, T_{0}\right) \\
& \text { Calculation of } f\left(x_{0}\right) \\
& \text { Until convergence } \\
& \text { Generation of new state } x_{1} \\
& \text { if } f\left(x_{1}\right)<f\left(x_{0}\right) \\
& x_{0}=x_{1} \\
& \text { else } \\
& \text { if exp }\left(\frac{f\left(x_{0}\right)-f\left(x_{1}\right)}{T}\right)>\operatorname{Rand}(0,1) \\
& \quad \text { Accept new solution } x_{0}=x_{1} \\
& \text { else } \\
& \text { Reject new solution } \\
& \text { Decrease the temperature } \\
& \text { end (Until) }
\end{aligned}
$$

At this moment we would like to introduce new simulated annealing method for optimal control of the multistage differential-algebraic systems. We start the presentation with some remarks.

Remark 1. The optimal control problem with index-1 differential-algebraic constraints using the direct shooting approach has been transformed into nonlinear optimization 
problem with equality constraints of the following form

$$
\begin{gathered}
\min _{x} \hat{Q}(x) \\
c_{\text {cons }}(x)=0 \\
c_{\text {cont }}(x)=0 .
\end{gathered}
$$

Remark 2. The quality $\mathcal{Q}$ of any proposed solution $x$ can be specified using a triple

$$
\mathcal{Q}(x)=\left[\begin{array}{l}
q_{1}(x) \\
q_{2}(x) \\
q_{3}(x)
\end{array}\right]=\left[\begin{array}{c}
\hat{Q}(x) \\
c_{\text {cons }}(x) \\
c_{\text {cont }}(x)
\end{array}\right]
$$

Remark 3. The "stretching" function technique applied for some proposed solution transforms the vector, which parametrized the quality of the proposed solution

$$
\mathcal{S}(\mathcal{Q}(x))=\mathcal{Q}^{\mathcal{S}}(x)=\left[\begin{array}{c}
q_{1}^{\mathcal{S}}(x) \\
q_{2}^{\mathcal{S}}(x) \\
q_{3}^{\mathcal{S}}(x)
\end{array}\right]=\left[\begin{array}{c}
\hat{Q}^{\mathcal{S}}(x) \\
c_{\text {cons }}^{\mathcal{S}}(x) \\
c_{\text {cont }}^{\mathcal{S}}(x)
\end{array}\right]
$$

The decisions of the new solution acceptance can be made by quality comparison of the considered solutions

Definition 4. Any two values $\mathcal{Q}_{a}$ and $\mathcal{Q}_{b}$ are in the relation $\oplus$ if

$$
\begin{aligned}
& q_{1}(a) \oplus q_{1}(b) \\
& q_{2}(a) \oplus q_{2}(b) \\
& q_{3}(a) \oplus q_{3}(b)
\end{aligned}
$$

Theorem 5. Let $x_{0}$ and $x_{1}$ be any two solutions. The solution $x_{0}$ is unconditionally better than solution $x_{1}$ if and only if

$$
\mathcal{Q}\left(x_{0}\right)<\mathcal{Q}\left(x_{1}\right) .
$$

Let us transform the triple, which defines the quality of the solution, using the "stretching" function technique.

Theorem 6. Let $x_{0}$ and $x_{1}$ be any two solutions. If

$$
\begin{aligned}
& q_{1}^{\mathcal{S}}\left(x_{0}\right)<q_{1}^{\mathcal{S}}\left(x_{1}\right) \\
& q_{2}^{\mathcal{S}}\left(x_{0}\right)<q_{2}^{\mathcal{S}}\left(x_{1}\right) \\
& q_{3}^{\mathcal{S}}\left(x_{0}\right)<q_{3}^{\mathcal{S}}\left(x_{1}\right)
\end{aligned}
$$

then

$$
\mathcal{Q}\left(x_{0}\right)<\mathcal{Q}\left(x_{1}\right) .
$$

The question is, how to compare two solutions, which are not in the relation presented in Theorem 6.

One of the most important features of simulated annealing algorithm is a possibility of accepting a step, which does not improve the current solution. Therefore, to compare any two solution, which are not in clear relation, a conditional acceptance mode can be applied. In simulated annealing methodology, it is known as an acceptance with probability. In the presented work, because of three-dimensional quality solution index $\mathcal{Q}(x)$ under considerations, a new extended method of acceptance with probability was proposed.

Proposition 7. Let $x_{0}$ be the current solution and $x_{1}$ be any other solution generated by simulated annealing algorithm from the neighborhood of the point $x_{0}$, and $T$ denote the value of the temperature in the current iteration. If the point $x_{1}$ cannot be unconditionally accepted as a new solution, then it can be accepted with the probability $p \in \mathcal{N}(0,1)$. The acceptance probability is dependent on the index quality in the following way

$$
\begin{array}{ll} 
& \exp \left(-\frac{\hat{Q}^{\mathcal{S}}\left(x_{1}\right)-\hat{Q}\left(x_{0}\right)}{T}\right)>p \\
\text { or } & \exp \left(-\frac{c_{\text {cons }}^{\mathcal{S}}\left(x_{1}\right)-c_{\text {cons }}\left(x_{0}\right)}{T}\right)>p \\
\text { or } & \exp \left(-\frac{c_{\text {cont }}^{\mathcal{S}}\left(x_{1}\right)-c_{\text {cont }}\left(x_{0}\right)}{T}\right)>p .
\end{array}
$$

There are two important remarks connected with the presented acceptance condition

Remark 8. The multiple conditions increase the general acceptance probability of the worse solution.

Remark 9. Application of the "stretching" function technique transforms the bad solution in the worse one. Moreover, "stretching" function technique supports searching for better solutions and alleviate the local minima.

The presented simulated annealing "stretching" function algorithm with new conditional solution acceptance approach was applied to solve the optimal control problem of the multistage rectors system.

\section{NUMERICAL EXAMPLES}

The presented methodology was tested on the optimization problem of the three-stage technological process. The presented system consists of two chemical reactors with mixing [24]. The sketch of the process was presented in the Fig. 1.

At the beginning, the first reactor was loaded with the substrate $A$ with the volume $0.1 \mathrm{~m}^{3}$ and concentration 2000 $\mathrm{mol} / \mathrm{m}^{3}$. Due to reactions taking place in the system, the products $B$ and $C$ are obtained according to the following scheme

$$
2 A \rightarrow B \rightarrow C .
$$

Additionally, the first reactor was equipped with a heating exchanger, which can be used to control the process temperature and in this way - to influence the trajectories of the process variables. The concentrations of the substrate and products were changing in the following way

$$
\begin{gathered}
\dot{C}_{A}=-2 k_{1}(T) C_{A}^{2} \\
\dot{C}_{B}=2 k_{1}(T) C_{A}^{2}-k_{2}(T) C_{B} \\
\dot{C}_{C}=k_{2}(T) C_{B}
\end{gathered}
$$

with the kinetics constraints

$$
k_{1}(T)=0.0444 \exp (-2500 / T)
$$

and

$$
k_{2}(T)=6889.0 \exp (-5000 / T) .
$$

Then, in the mixing stage at the time $t_{1}$, the component $B$ of concentration $C_{B}^{0}=600 \mathrm{~mol} / \mathrm{m}^{3}$ and some volume $S$ was added. Therefore, the volume and consecrations of the substrates were changing, so the following relations were satisfied

$$
\begin{gathered}
V_{2} C_{A}\left(t_{2}^{0}\right)=V_{1} C_{A}\left(t_{1}\right) \\
V_{2} C_{B}\left(t_{2}^{0}\right)=V_{1} C_{B}\left(t_{1}\right)+S C_{B}^{0}
\end{gathered}
$$




$$
V_{2} C_{C}\left(t_{2}^{0}\right)=V_{1} C_{C}\left(t_{1}\right)
$$

where $V_{1}$ is the volume of substrates loaded at the beginning of the first reactor. Therefore, the volume $V_{2}$ in the second reactor was given by

$$
V_{2}=V_{1}+S
$$

The volume $S$ is a decision parameter with

$$
0 \leq S \leq 0.1\left(m^{3}\right)
$$

After the mixing stage, the substrates were loaded into the last reactor, where three reactions were taking a place

$$
\begin{gathered}
B \rightarrow D \\
B \rightarrow E \\
2 B \rightarrow F
\end{gathered}
$$

In the 2 nd reactor, the reactions take place under isotherma conditions. The state variables are changing in the following way

$$
\begin{gathered}
\dot{C}_{A}=0 \\
\dot{C}_{B}=-0.02 C_{B}-0.05 C_{B}-2 \times 4.0 \times 10^{-5} C_{B}^{2} \\
\dot{C}_{C}=0 \\
\dot{C}_{D}=0.02 C_{B} \\
\dot{C}_{E}=0.05 C_{B} \\
\dot{C}_{F}=4.0 \times 10^{-5} C_{B}^{2}
\end{gathered}
$$

The combined processing time for both reactors is equal to $180 \mathrm{~min}$

$$
\begin{gathered}
t_{1}+t_{2}=180, \\
t_{1}>0, \quad t_{2}>0 .
\end{gathered}
$$

The decision variables are the profile of the temperature $T(t)$, the duration time of the reactions in each stage, and the amount $S$ of component $B$, which is added at the mixing step.

The process is aimed to maximize the amount of the product $D$ at the output of the $2 n d$ reactor

$$
\max _{t_{1}, t_{2}, S, T(t)} V_{2} C_{D}\left(t_{2}\right)
$$

subject to the constraints on the temperature profile

$$
298 \leq T(t) \leq 398(K), \quad t \in\left[\begin{array}{ll}
0 & t_{1}
\end{array}\right] .
$$

The direct shooting method enables us to transform the multistage optimal control problem as an nonlinear optimization problem with both continuous and pointwise constraints. In classical approaches, the nonlinear optimization problem with constraints can be solved using penalty function approach or NLP algorithms, like Sequential Quadratic Programming or Barrier methods. In this way, the process in first reactor was divided into 10 equidistance intervals. Additionally, the initial conditions for the second reactor should be consistent with the results of the mixing stage. Therefore, the considered NLP

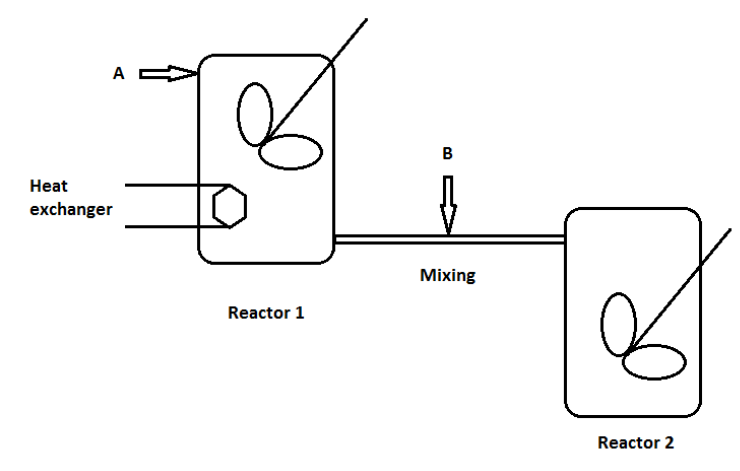

Fig. 1. System of the two-reactors with mixing.

problem consisted of 27 continuity constraints, 3 consistency constraints and 42 decision variables.

The aggregated model was defined using 3 scalar functions

$$
\begin{gathered}
\hat{Q}(x)=Q(x) \\
c_{\text {cont }}(x)=\sum_{i=1}^{27}\left\|c_{\text {cont }, 1}(x)\right\|_{1} \\
c_{\text {cons }}(x)=\sum_{i=1}^{3}\left\|c_{\text {cons }, 1}(x)\right\|_{1}
\end{gathered}
$$

In the next step, all three function were transformed using "stretching" function technique

$$
\begin{gathered}
\mathcal{S}(\hat{Q}(x))=\hat{Q}^{\mathcal{S}}(x) \\
\mathcal{S}\left(c_{\text {cont }}(x)\right)=c_{\text {cont }}^{\mathcal{S}}(x) \\
\mathcal{S}\left(c_{\text {cons }}(x)\right)=c_{\text {cons }}^{\mathcal{S}}(x)
\end{gathered}
$$

and optimized using presented simulated annealing algorithm with new acceptance method.

The parameters of the methods were adjusted automatically. Therefore, the simulations were performed with the following parameters

- number iterations in the outer loop MaxIter $=500$,

- inner iterations in cooling loop MaxIterCool $=10$,

- diameter of the considered neighborhood $\alpha=0.4$

- initial temperature $T_{0}=100$,

- temperature update factor $T_{i+1}=\beta T_{i}$ is $\beta=0.6$.

The obtained results, which were obtained for the aggregated model, were applied to the original model of the considered process. After 702 model evaluations, the final value of the original process performance index was equal to $22.7233 \mathrm{~mol}$. The obtained state trajectories were presented in the Fig. 2. 


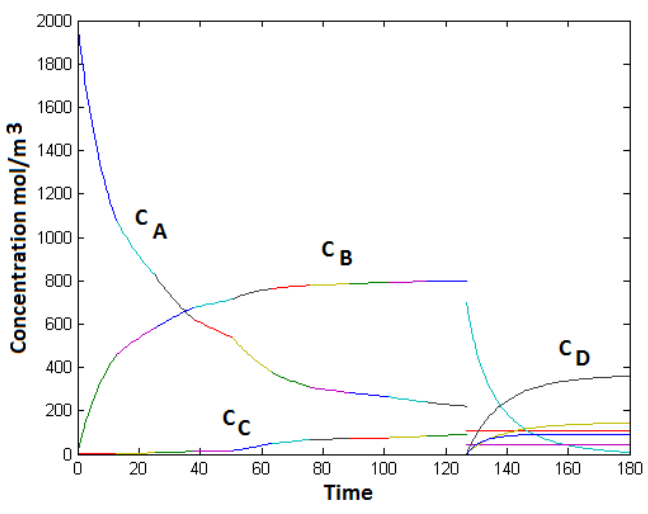

Fig. 2. Trajectories of the state variables.

\section{CONCLUSION}

In the article a problem of solving complex multistage technological processes was considered. The new methodology, which was presented, applies aggregation-disaggregation approach. The optimal control problem was transformed into the nonlinear optimization problem using the direct shooting approach. Then, the NLP problem has been defined using only three functions, which reflects the characteristics features of the process. The first one denotes the value of the process performance index. Then, the second one is connected with the continuity of the differential state trajectories, and the last one denotes the consistent initial conditions of the process. The quality of the proposed solution was defined using only values of these three functions. To compare any two proposed solutions, the new decision approach was proposed. The presented methodology was applied to the two-stage reactor system.

The future research will be devoted to error analysis of the aggregation-disaggregation procedures for control and optimization of the multistage technological processes with nonlinear differential-algebraic constraints.

\section{ACKNOWLEDGMENT}

Many thanks are due to two anonymous referees, whose constructive suggestions led to improvement of the original article.

The project was supported by the grant Młoda Kadra B40099/I6 at Wrocław University of Technology.

\section{REFERENCES}

[1] J.R. Banga, E. Balsa-Canto, C.G. Moles, A.A. Alonso. 2005. Dynamic optimization of bioprocesses: Efficient and robust numerical strategies. Journal of Biotechnology. 117:407-419, http://dx.doi.org/10.1016/j.jbiotec.2005.02.013

[2] J.T. Betts. 2010. Practical Methods for Optimal Control and Estimation Using Nonlinear Programming, Second Edition. SIAM, Philadelphia, http://dx.doi.org/10.1137/1.9780898718577

[3] L.T. Biegler. 2010. Nonlinear Programming. Concepts, Algorithms and Applications to Chemical Processes. SIAM, Philadelphia, http://dx.doi.org/10.1137/1.9780898719383
[4] L.T. Bielger, S. Campbell, V. Mehrmann. 2012. DAEs, Control, and Optimization. Control and Optimization with Differential-Algebraic Constraints. SIAM, Philadelphia, http://dx.doi.org/10.1137/9781611972252.ch1

[5] K.F. Bloss, L.T. Biegler, W.E. Schiesser. 1999. Dynamic process optimization through adjoint formulations and constraint aggregation. Ind. Eng. Chem. Res. 38:421-432, http://dx.doi.org/10.1021/ie9804733

[6] K.E. Brenan, S.L. Campbell, L.R. Petzold. 1996. Numerical Solution of Initial- Value Problems in Differential-Algebraic Equations. SIAM, Philadelphia, http://dx.doi.org/10.1137/1.9781611971224

[7] M. Diehl, H.G. Bock, J.P. Schlöder, R. Findeisen, Z. Nagy, F. Allgöwer. 2002. Real-time optimization and nonlinear model predictive control of processes governed by differential-algebraic equations. Journal of Process Control. 12:577-585, http://dx.doi.org/10.1016/S0959-1524(01)00023-3

[8] P. Drạg, K. Styczeń. 2012. A Two-Step Approach for Optimal Control of Kinetic Batch Reactor with electroneutrality condition. Przeglad Elektrotechniczny. 6:176-180.

[9] S. El Moumen, R. Ellaia, R. Aboulaich. 2011. A new hybrid method for solving global optimization problem. Applied Mathematics and Computation. 218:3265-3276, http://dx.doi.org/10.1016/j.amc.2011.08.066

[10] R. Faber, T. Jockenhövel, G. Tsatsaronis. 2005. Dynamic optimization with simulated annealing. Computers and Chemical Engineering. 29:273290, http://dx.doi.org/10.1016/j.compchemeng.2004.08.020

[11] S. Fidanova, M. Paprzycki, O. Roeva. 2014. Hybrid GA-ACO Algorithm for a Model Parameters Identification Problem. 2014 Federated Conference on Computer Science and Information Systems. 413-420, http://dx.doi.org/10.15439/2014F373

[12] R. Fletcher, S. Leyffer. 2002. Nonlinear programming without a penalty function. Mathematical Programming. 91:239-269, http://dx.doi.org/10.1007/s101070100244

[13] M. Gerdts. 2003. Direct Shooting Method for the Numerical Solution of Higher-Index DAE Optimal Control Problems. Journal of Optimization Theory and Applications. 117:267-294, http://dx.doi.org/10.1023/A:1023679622905

[14] M. Jeon. 2005. Parallel optimal control with multiple shooting, constraints aggregation and adjoint methods. J. Appl. Math. and Computing 19:215-229, http://dx.doi.org/10.1007/BF02935800

[15] M. Ji, Z. Jin, H. Tang. 2006. An improved simulated annealing for solving the linear constrained optimization problems. Applied Mathematics and Computation. 183:251-259, doi:10.1016/j.amc.2006.05.070

[16] M. Kwiatkowska. 2015. DAEs method for time-varying indoor air parameters evaluation. In: A. Kotowski, K. Piekarska, B. Kaźmierczak (eds.) Interdyscyplinarne zagadnienia w inżynierii i ochronie środowiska T. 6. Wrocław 2015, pp. 214-220.

[17] D.B. Leineweber, I. Bauer, H.G. Bock, J.P. Schlöder. 2003. An efficient multiple shooting based reduced SQP strategy for large-scale dynamic process optimization. Part 1: theoretical aspects. Computer and Chemical Engineering. 27:157-166, http://dx.doi.org/10.1016/S00981354(02)00158-8

[18] J. Nocedal, S.J. Wright. 2006. Numerical Optimization. Second Edition. Springer, New York, http://dx.doi.org/10.1007/978-0-387-40065-5

[19] K.E. Parsopoulos, V.P. Plagianakos, G.D. Mogoulas, M.N. Vrahatis. 2001. Improving the particle swarm optimizer by function "stretching", in: N. Hadjisavvas, P.M. Pardalos (eds.), Advances in Convex Analysis and Global Optimization, Kluwer Academic Publishers. 445-457, http://dx.doi.org/10.1007/978-1-4613-0279-7_28

[20] K.E. Parsopoulos, V.P. Plagianakos, G.D. Mogoulas, M.N. Vrahatis. 2001. Objective function "stretching" to alleviate convergence to local minima. Nonlinear Analysis. 47:3419-3424, http://dx.doi.org/10.1016/S0362-546X(01)00457-6

[21] K.E. Parsopoulos, M.N. Vrahatis. 2002. Particle Swarm Optimization Method for Constrained Optimization Problems, in: P. Sincak et al.(Eds.) Intelligent Technologies - Theory and Applications: New Trends in Intelligent Technologies, IOS Press, pp. 214-220.

[22] E. Rafajłowicz, K. Styczeń, W. Rafajłowicz. 2012. A modified filter SQP method as a tool for optimal control of nonlinear systems with spatio-temporal dynamics. Int. J. Appl. Math. Comput. Sci. 22:313-326, http://dx.doi.org/10.2478/v10006-012-0023-8

[23] D.F. Rogers, R.D. Plante, R.T. Wong, J.R. Evans. 1991. Aggregation and disaggregation techniques and methodology in optimization. Operations Research. 39:553-582, http://dx.doi.org/10.1287/opre.39.4.553

[24] V.S Vassiliadis, R.W.H. Sargent, C.C. Pantelides. 1994. Solution of a Class of Multistage Dynamic Optimization Problems. 1. Prob- 
lems without Path Constraints. Ind. Eng. Chem. Res. 33:2111-2122, http://dx.doi.org/10.1021/ie00033a014

[25] Y.-J. Wang, J.-S. Zhang 2007. An efficient algorithm for large scale global optimization of continuous functions. Journal of Computational and Applied Mathematics. 206:1015-1026, http://dx.doi.org/10.1016/j.cam.2006.09.006

[26] K.F.C. Yiu, Y. Liu, K.L. Teo. 2004. A hybrid descent method for global optimization. Journal of Global Optimization. 28:229-238, http://dx.doi.org/10.1023/B:JOGO.0000015313.93974.b0 